\title{
EXCITONS IN TERNARY MIXED MOLECULAR CRYSTALS: A PROTOTYPE FOR THE PRIMARY STEP OF PHOTOSYNTHESIS? *
}

\author{
Raoul KOPELMAN \\ Department of Chemistry, The University of Michigan, \\ Ann Arbor, Michigan 48104, USA
}

\begin{abstract}
Exciton percolation, which has been demonstrated in synthetic ternary molecular alloys, is applied to photosynthetic light harvesting complexes. The resulting model ("substitutionally random") results in a predicted pigment $A / B$ ratio larger than one, where $A$ represents the lowest energy pigment species and $B$ the higher substitutional ones (i.e. chl $\mathrm{a} / \mathrm{chl} \mathrm{b}$ in plants). An additional criterion is given for the nature of the reaction center (i.e. P700), based on phonon amalgamation with species A (i.e. structural similarity).
\end{abstract}

\section{Introduction}

The concept of exciton percolation has been discussed in a preliminary fashion recently $[1-4]$. In a randomly substituted binary mixed crystal, where the excitation energy is directly or indirectly injected into the lower energy component, there appears a critical concentration at which this molecular alloy effectively undergoes an exciton insulator-to-exciton conductor transition (with a small change in a parameter which may be concentration, pressure, temperature, etc.). To monitor this behaviour we added a third component (sensor or supertrap) to measure the exciton "conduction". Preliminary experiments have been described [3-5].

Our model systems used for the demonstration of exciton percolation (say naphthalene/perdeuteronnaphthalene: betamethylnaphthalene) bear a striking resemblance to certain natural photosynthetic systems (say chlorophyll a/chlorophyll b: P700). This led us to investigate a substitutionally random model for the light harvesting complex in photosynthetic systems like those in green plants.

\section{The model}

Consider a set $\mathrm{A}$ of molecules (sites) with excitation energy $E_{\mathrm{A}}$ (with a "smear" $\Delta E_{\mathrm{A}}<k T$, where $k$ is the Boltzmann constant and $T$ the absolute temperature),

* Supported by NIH Grant NS08116-08 and NSF Grant GH-32578X. 
called the energy conducting species. Consider another set B, with excitation energy $E_{\mathrm{B}}$, where

$$
E_{\mathrm{B}}-E_{\mathrm{A}}>k T .
$$

This set is called the energy insulating species (the smear $\Delta E_{\mathrm{B}}$ can be large, as long as eq. (1) is obeyed). The third set, $\mathrm{S}$, has excitation energies $E_{\mathrm{S}}$, where

$$
E_{\mathrm{A}}-E_{\mathrm{S}}>k T,
$$

and is called the sensor or supertrap species (the smear $\Delta E_{\mathrm{S}}$ can again be large, as long as eq. (2) is obeyed).

The above three species form one lattice of sites (substitutional sites), randomly distributed, with a given topology of interactions. The interaction topology involves the numbers and arrangements of the physically interacting neighbors (i.e. 4 nearest neighbor in-plane interactions with fourfold symmetry, with an option of two additional interactions in the same plane with twofold symmetry, with an option of two more out-of-plane ones with inversion symmetry, etc.).

In our present model we limit the molar concentration $C_{\mathrm{S}}$ of the $\mathrm{S}$ sites to a small number, of the order of $1 \%$ or less, such that

$$
C_{\mathrm{S}} \ll C_{\mathrm{A}} .
$$

The important parameter now is $C_{\mathrm{A}}$, or alternatively the ratio

$$
R_{\mathrm{A}} \equiv C_{\mathrm{A}} / C_{\mathrm{B}} .
$$

We now proceed on the assumption that the energy conduction is confined to the quasilattice of set $\mathrm{A}$ and depends critically on its connectivity. This connectivity depends critically on the concentration $C_{\mathrm{A}}$, or alternatively, the concentration ratio $R_{\mathrm{A}}$. The quantitative relationships are based on percolation theory. In the limit where the energy conduction in a pure A lattice (with the same topology) is very efficient the energy conduction in our substitutionally random lattice is proportional to $\overline{P_{\infty} \mathrm{A}}$, the probability of an $\mathrm{A}$ site being inside the $\mathrm{A}$ maxi-cluster (the largest $\mathrm{A}$ cluster) $[3,6,7]$. This probability is essentially zero for $0 \leqslant C_{\mathrm{A}}<C_{\mathrm{c}}$, where $C_{\mathrm{c}}$ is the critical percolation concentration (i.e. 0.59 for a square lattice, 0.50 for a triangular one, 0.31 for a simple cubic one, etc.). At $C_{\mathrm{c}}$ the value of $\bar{P}_{\infty}^{\mathrm{A}}$ climbs fast and approaches 0.99 for $C_{\mathrm{A}} \approx C_{\mathrm{c}}+0.15$. This behaviour is shown in fig. 1 . Thus the condition for energy transfer is

$$
R_{\mathrm{A}}>1 \quad \text { (within a factor of 2). }
$$




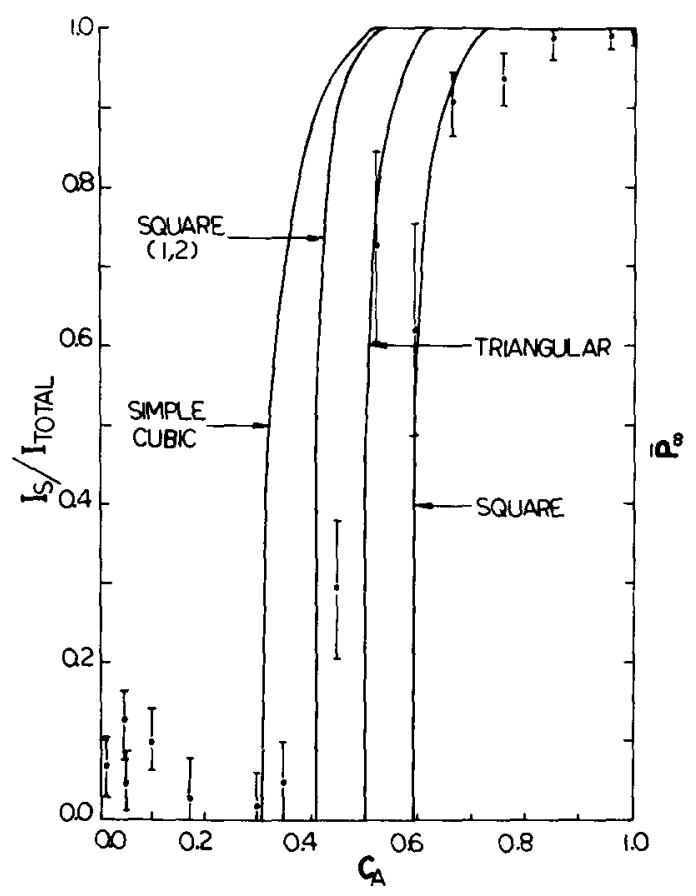

Fig. 1. Theoretical and experimental exciton percolation curves. The theoretical square lattice site percolation $\bar{P}_{\infty}$ curve (for component A) is the same as in ref. [3] (except for a different normalization: $P_{\infty}=C_{\mathrm{A}} \bar{P}_{\infty}$ ), while "SQUARE $(1,2)$ ", for the square lattice with both nearest and next-nearest neighbor interactions (coordination number 8 ), is based on ref. [8]. The triangular (coordination number 6 ) and simple cubic curves are from H.L. Frish, J.M. Hammersley and D.J.A. Welch, Phys. Rev. 126 (1962) 949. The experimental points are integrated fluorescence intensity ratios for the first singlet excitation of naphthalene (A) in perdeuteronaphthalene (B) with a sensor of betamethylnaphthalene (S) and are further described in ref. [3].

To emphasize this result, we write an equivalent relationship:

$$
0 \leqslant R_{\mathrm{B}} \equiv C_{\mathrm{B}} / C_{\mathrm{A}}<1 \pm 0.5
$$

\section{Discussion}

Our very general condition on energy transfer (eq. (6)) is independent of whether the transfer is a coherent exciton transfer or a hopping exciton transfer (equivalent to resonant Dexter-Foster transfer). It will be affected if instead of a perfectly random distribution one has partial aggregation tendencies (A with either A or B), but not significantly, as long as there is no complete segregation. Notice that any "segregation model" (or "funnel model") requires some kind of surrounding of the $\mathrm{S}$ 
species by the A species and some partial surrounding of the latter by the B species, in some ordered or semi-ordered fashion (i.e. an "energy funnel"). Note also that in our model an occasional thermalization of the excitation, out of the supertrap, will easily result in its trapping by a different supertrap, while this kind of "spillover" is not at all likely in the funnel model. However, the main distinction between our quasi-random model and the semi-ordered one is that in the latter there is no restriction on the ratio $R_{\mathrm{A}}$, i.e.

$$
\begin{aligned}
& 0 \leqslant R_{\mathrm{A}}=R_{\mathrm{B}}^{-1} \leqslant \infty, \\
& 0 \leqslant R_{\mathrm{B}}=R_{\mathrm{A}}^{-1} \leqslant \infty .
\end{aligned}
$$

The only time when eqs. (7), (8) are valid in a random system is when the excitoninteractions are extremely long range. An example for this is the long-range superexchange (tunneling) interaction demonstrated by the extremely long-lived triplet states of aromatic crystals (i.e. $R_{\mathrm{A}} \approx 0.1$ for naphthalene [4]). We notice however, that the superexchange process is efficient only for an energetically homogeneous host (i.e. $\Delta E_{\mathrm{B}} \rightarrow 0$ ). On the other hand, when the excitation transfer is extremely inefficient (i.e. for a very short lifetime and/or very low sensor concentration and/or trapping efficiency), one can get into the regime where efficient energy transfer occurs only for

$$
R_{\mathrm{A}} \gg 1 \text {. }
$$

Our computer simulations $[7,8]$ show that the above principles hold for twodimensional lattices as small as $20 \times 20$ as well as for three-dimensional lattices with an Avogadro (or infinite) number of sites (with only minor variation with boundary conditions). Obviously, on the other hand, for a one-dimensional-lattice, $C_{\mathrm{c}}=1$ and thus for one-dimensional energy transfer,

$$
R_{\mathrm{A}} \rightarrow \infty
$$

i.e. no substitutional $B$ sites are allowed (note that interstitial $B$ species have been excluded from our discussion and should not be counted towards the $R$ ratios!).

An additional point common to our synthetic molecular alloys and to the photosynthetic systems is the requirement that the sensor (supertrap, active center), which is a definite minority component, should be a very efficient exciton trap. This requires it to have an amalgamated phonon band [9-12] with the energy conducting species $(A)$, in contrast to localized phonons [13]. The requirements for an amalgamated (delocalized) phonon band in the synthetic alloys [14] boil down to a similarity in chemical shape (giving similar masses, moments of inertia, van der Waals packing sizes and intermolecular force constants). This requirement is fulfilled in the photosynthetic unit provided that the active center (i.e. P700) is essentially the same 
molecule as the energy conducting species (i.e. chlorophyll a), except for minor changes (being part of a hydrate and/or dimer and/or special aggregate). This "phonon criterion" is independent of percolation or the random model and will be discussed further elsewhere.

\section{References}

[1] H-K. Hong and R. Kopelman, J. Chem. Phys. 55 (1971) 5380.

[2] R. Kopelman, in: Excited states, Vol. II, ed. E. Lim (Academic Press, New York, 1975).

[3] R. Kopelman, E.M. Monberg, F.W. Ochs and P.N. Prasad, Phys. Rev, Letters 34 (1975) 1506.

[4] R. Kopelman, E.M. Monberg, F.W. Ochs and P.N. Prasad, J. Chem. Phys. 62 (1975) 292.

[5] F.W. Ochs, Ph. D. Thesis, University of Michigan (1974).

[6] S. Kirkpatrick, Rev. Mod. Phys. 45 (1973) 574, and references therein.

[7] J. Hoshen and R. Kopelman, unpublished.

[8] P. Argyrakis, J. Hoshen and R. Kopelman, unpublished.

[9] P.N. Prasad and R. Kopelman, J. Chem. Phys. 57 (1972) 863.

[10] R. Kopelman, F.W. Ochs and P.N. Prasad, J. Chem. Phys. 57 (1972) 5409.

[11] H.-K. Hong and R. Kopelman, J. Chem. Phys. 58 (1973) 2557.

[12] H.-K. Hong and R. Kopelman, J. Chem. Phys. 58 (1973) 384.

[13] P.H. Chereson, P.S. Friedman and R. Kopelman, J. Chem. Phys. 56 (1972) 3716.

[14] P. Argyrakis, E.M. Monberg and R. Kopelman, unpublished.

\section{Discussion}

D. Haarer: Percolation can be looked at in a geometrical, i.e. time independent way. Did you also look into the dynamics, i.e. coherent or incoherent exciton transfer?

R. Kopelman: Yes. The dynamics are very strongly affected by the topology. We have calculated transfer curves as a function of guest concentration, sensor concentration, topology, exciton coherence length (anisotropic with crystal directions), coherence time, trapping efficiency, exciton lifetime, jump time, etc.

H. Killesreiter: In your model you give emphasis to the distance between adjacent molecules. Did you also take care of the relative orientations that are very essential for energy interactions?

R. Kopelman: Yes. First these are included in our topology, implicitly. Furthermore, in our dynamical investigations (see my answer to Dr. D. Haarer) we have explicitly used pair-wise exciton interactions which are definitely a function of relative orientation (see H.K. Hong and R. Kopelman, J. Chem. Phys. 55 (1971) 724; F.W. Ochs, P.N. Prasad and R. Kopelman, Chem. Phys. 6 (1974) 253).

H.C. Wolf: In order to understand the relative intensities of host, guest and supertrap emission one should take into account that the guest emission from naph thalene- $h_{8}$ is a superposition of emissions from different species with different trap depth. The result is a competition between several different "supertraps" which depends both on temperature and concentration.

R. Kopel man: Yes. We obviously observe this in our own spectra as well (see also P. Argyrakis, E.M. Monberg and R. Kopelman, Chem. Phys. Letters 36 (1975) 349). However, the way we define the energy transfer and also the way we monitor it experimentally, using a sensor (supertrap), is essentially independent of the "internal" competition among the guest (naphthalene) clusters (as long as tunneling and temperature activation are negligible). 
M. Schott: It is often felt that, among different possibilities, a living system "choses" to operate according to the most efficient, the other being eliminated by selection pressures. Could you comment on the advantages, for the green plants, of using, in photosynthesis, percolation in a random mixture as you prefer, rather than the "funnel" structure?

R. Kopelman: Yes, I can speculate. One advantage is that it minimizes the effects of impurities, defects and domain boundaries on the energy transfer rate (this is also true for molecular alloys versus pure crystals). It may be difficult for nature to produce large antenna aggregates that are free of such defects. This is one advantage of the "random substitution" model. Another advantage is that it allows for much easier "spill-over" from one active center to another; some biologists also believe that such a model is more adaptable to variations in growing conditions (like light availability). 